\title{
(C) OPEN ACCESS \\ Patient-reported complications related to peripherally inserted central catheters: a multicentre prospective cohort study
}

\author{
Sarah L Krein, ${ }^{1,2}$ Sanjay Saint, ${ }^{1,3}$ Barbara W Trautner, ${ }^{4,5}$ Latoya Kuhn, $^{1}$ \\ John Colozzi, ${ }^{1}$ David Ratz, ${ }^{1}$ Erica Lescinskas, ${ }^{5}$ Vineet Chopra ${ }^{1,3}$
}

- Additional material is published online only. To view please visit the journal online (http://dx.doi.org/10.1136/ bmjqs-2018-008726).

For numbered affiliations see end of article.

\section{Correspondence to} Dr Sarah L Krein, Center for Clinical Management Research, VA Ann Arbor Healthcare System, Ann Arbor, MI 48105, USA; skrein@umich.edu

Received 16 August 2018 Revised 6 November 2018 Accepted 11 November 2018 Published Online First 25 January 2019
Check for updates

(C) Author(s) (or their employer(s)) 2019. Re-use permitted under CC BY-NC. No commercial re-use. See rights and permissions. Published by BMJ.

To cite: Krein SL, Saint S,

Trautner BW, et al.

BMJ Qual Saf

2019:28:574-581.

\section{ABSTRACT}

Objective Peripherally inserted central catheters (PICC) are frequently used to deliver medical therapies, but our knowledge regarding PICC-related complications remains incomplete. The objective of this study was to systematically elicit and characterise PICC-related complications as experienced by patients during and after hospitalisation.

Design Prospective cohort study.

Setting Inpatient medical units at four US hospitals in two states.

Participants Consecutive sample of patients who had a new PICC placed during a hospital stay between August 2015 and May 2017.

Main outcome(s) Patient-reported signs and symptoms of a possible PICC-related complication or functional issues.

Results of the 438 patients in the analytic cohort (91.4\% of those consented), two-thirds were male with a mean age of 56 years. The most common reason for PICC placement was long-term antibiotic therapy (43.4\%). During the 70 -day follow-up period, $61.4 \%$ of patients reported signs of at least one complication, including potentially serious complications, such as bloodstream infection (17.6\%) and deep vein thrombosis (30.6\%). Correspondence of these reported events with medical record documentation of the complication was generally low. More than one-quarter (27.9\%) of patients reported minor complications, such as insertion site redness, discomfort or difficult removal. While the PICC was in place, $26.0 \%$ reported restrictions in activities of daily living, $14.4 \%$ social activity restrictions and $19.2 \%$ had difficulty with flushing or operating the PICC.

Conclusion Over $60 \%$ of patients report signs or symptoms of a possible complication or adverse effect after PICC placement. Bothersome complications from the patient perspective are clearly more common than those that typically rise to the level of healthcare provider attention or concern. Understanding the patient experience is critical for providing safe and effective care.

\section{INTRODUCTION}

Since their development in the 1970 s, ${ }^{12}$ peripherally inserted central catheters (PICC) have become an integral part of modern healthcare. PICCs are frequently inserted in hospitalised patients to maintain venous access and facilitate administration of vital therapies, such as antibiotics and chemotherapy. ${ }^{34}$ Moreover, PICCs allow patients to receive these therapies outside the hospital setting, enabling early discharge and transitions of care to alternative settings.

The usefulness of PICCs for care delivery is clear. Yet, concerns about inappropriate use and the consequent potential for complications and preventable patient harm have been raised. ${ }^{56}$ As with any invasive medical device, PICCs are associated with serious complications, including central line-associated bloodstream infection (CLABSI) and venous thromboembolism. ${ }^{7-9}$ One recent study, for example, found considerable variability in PICC use and indications for use as well as PICC-related complications across a sample of 10 hospitals. ${ }^{10}$ Such findings have stimulated increased focus on ensuring appropriate PICC use in hospitalised patients, including Choosing Wisely recommendations for these devices. ${ }^{6}$

Unfortunately, our knowledge regarding PICC-related complications remains incomplete. Although PICCs are often inserted during hospitalisation, they facilitate intravenous treatment outside of this monitored setting. ${ }^{41}$ Despite this, little attention has been paid to complications as experienced by patients following PICC placement and especially after hospital discharge. ${ }^{12} 13$ Several qualitative studies have explored specific patient experiences, such as quality of life among patients with cancer ${ }^{14}$ or after a symptomatic deep vein thrombosis (DVT), ${ }^{15}$ and the impact of arm choice on daily 


\section{Patients identified as potentially eligible}

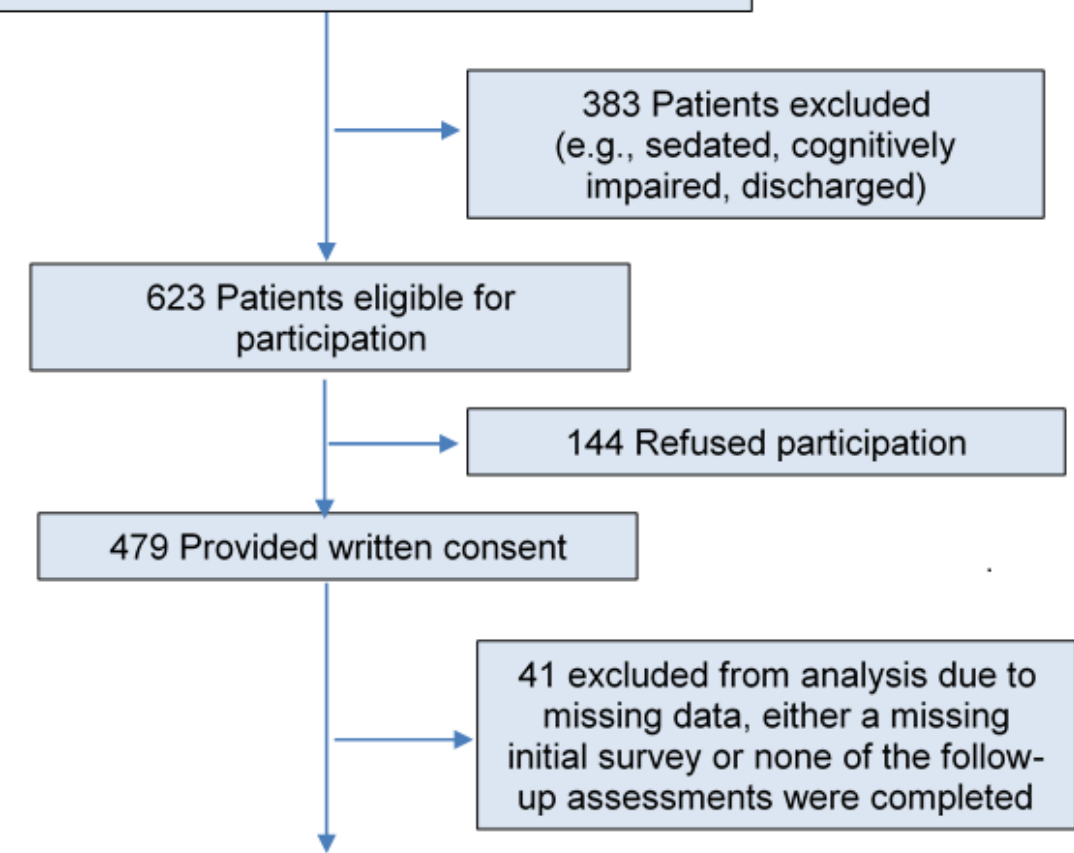

\section{Provided baseline and at least 1 follow-up assessment 341 completed 14-day follow-up 379 completed 30-day follow-up 357 completed 70-day follow-up}

Figure 1 Study enrolment and follow-up. In total, 1006 patients were initially identified as potentially eligible to participate in the study from the four participating study sites. Of the 623 who met eligibility criteria, $479(77 \%)$ agreed to participate. Those who did not complete at least one follow-up assessment $(n=41)$ were excluded from this analysis due to missing data.

activities. ${ }^{16}$ On the other hand, issues such as frequency of minor complications (such as insertion site redness), how PICCs impact daily activities and other concerns that might arise posthospitalisation have not been fully examined. Understanding these issues from the patient perspective is a critical first step for identifying PICC-associated patient concerns, which could result in both warranted and unwarranted healthcare utilisation, and for making care more patient centred. Thus, we sought to systematically elicit and characterise patient-reported concerns to provide a more comprehensive picture of PICC use and potential complications as experienced by patients during and beyond hospitalisation.

\section{METHODS}

\section{Study overview}

We conducted a prospective observational study involving patients receiving care at four US hospitals. From August 2015 through May 2017, patients who had a PICC inserted for clinical care during a hospital admission were recruited and followed for 70 days from the date of initial PICC placement.
Follow-up continued regardless of whether the PICC was removed, exchanged or replaced. Information about potential complications was collected through patient self-report within 3 days of initial PICC placement and then at 14, 30 and 70 days using a standardised questionnaire. Selected data elements were also collected through a medical record review conducted by research staff at each site. Participating hospitals included two Veterans Affairs medical centres and two academic centres in Michigan and Texas.

\section{Patient recruitment and data collection}

Each weekday morning (Monday through Friday) research staff used electronic medical records or lists provided by PICC inserters to identify patients who received a PICC. Research staff then visited patient rooms to confirm eligibility. Potentially eligible patients included those who: (1) were at least 18 years of age; (2) were hospitalised on an acute care unit, including medical/surgical wards, intensive care and progressive care units; (3) had a PICC that was placed for the first time during the hospital stay (ie, new insertion that was in place for $\leq 3$ days); and (4) spoke either English 
Table 1 Baseline patient and PICC characteristics $(n=438)$

\begin{tabular}{|c|c|}
\hline Characteristic & n $(\%)$ \\
\hline Age in years, mean (SD) & $56.4 \pm 15.6$ \\
\hline \multicolumn{2}{|l|}{ Sex } \\
\hline Male & $294(67.1)$ \\
\hline Female & $144(32.9)$ \\
\hline \multicolumn{2}{|l|}{ Race } \\
\hline White & $347(79.2)$ \\
\hline Black & $66(15.1)$ \\
\hline $\begin{array}{l}\text { Other (eg, Asian, American Indian, prefer not to } \\
\text { answer) }\end{array}$ & $25(5.7)$ \\
\hline Hispanic & $45(10.3)$ \\
\hline \multicolumn{2}{|l|}{ Patient-reported indication for placement } \\
\hline Long-term antibiotics & $190(43.4)$ \\
\hline Difficult venous access & $72(16.4)$ \\
\hline Chemotherapy & $90(20.5)$ \\
\hline Total parenteral nutrition (TPN) & $31(7.1)$ \\
\hline Other or unknown (eg, need medications) & $55(12.6)$ \\
\hline Device placed by vascular access/PICC team nurse & $347(79.2)$ \\
\hline More than one placement attempt & $74(16.9)$ \\
\hline $\begin{array}{l}\text { Experienced pain, discomfort, bleeding or other trauma } \\
\text { during insertion }\end{array}$ & $129(29.5)$ \\
\hline \multicolumn{2}{|l|}{ Number of devices during 70-day follow-up period* } \\
\hline 1 & $357(81.5)$ \\
\hline 2 & $45(10.3)$ \\
\hline$\geq 3$ & $36(8.2)$ \\
\hline \multicolumn{2}{|l|}{ Initial device, dwell time* (days) } \\
\hline$\leq 5$ & $107(24.4)$ \\
\hline $6-14$ & $118(26.9)$ \\
\hline $15-30$ & $72(16.4)$ \\
\hline$>30$ & $132(30.1)$ \\
\hline Unknown & $9(2.1)$ \\
\hline \multicolumn{2}{|l|}{ Initial device, number of lumens* } \\
\hline 1 & $183(41.8)$ \\
\hline 2 & $207(47.3)$ \\
\hline 3 & $18(4.1)$ \\
\hline Unknown & $30(6.8)$ \\
\hline
\end{tabular}

PICC, peripherally inserted central catheter.

*Information derived primarily from chart review data.

or Spanish. Exclusion criteria were: (1) did not have the capacity to provide self-consent or participate in the interview/assessment process (eg, obtunded, severe dementia, delirium); (2) refused to provide written informed consent to participate; or (3) had previously participated in this project. Patients deemed eligible based on these criteria were invited to participate in the study.

After a patient agreed to participate, research staff obtained informed consent and conducted a brief structured in-person interview. Those who chose to participate also received a magnet (either in the shape of the state in which they were recruited or branch of military in which they served) as a thank you for their participation. The initial interview included general demographic questions as well as questions about the
PICC insertion process, including reason for PICC insertion, type of professional who placed the device, number of insertion attempts and whether the patient experienced any pain/discomfort/bleeding/other trauma during placement. Follow-up assessments were conducted in person if the participant was still hospitalised or by telephone if discharged at approximately 14, 30 and 70 days after initial PICC insertion. These assessments consisted of a structured set of questions to first ascertain if the PICC was still in place or had been removed, and if removed, whether another PICC had been placed. Next, patients responded to a series of yes/no questions about signs or symptoms (during the past 7 days at the 14-day call and past 30 days at the 30 and 70 -day calls) that might indicate a potential PICC-related complication.

Specific signs or symptoms of a potential medical complication for which participants were queried included the following: site redness; redness, pain or swelling in the arm or shoulder; chest pain, shortness of breath or difficulty breathing that required emergency care; pain, swelling, redness in legs or calves; and fevers, chills or symptoms suggestive of an infection that required them to see a doctor. We asked about pain, swelling or redness in legs or calves because PICC use has been associated with lower extremity DVT. ${ }^{17}$ Patients who no longer had a PICC in place were also asked whether they were told they had a bloodstream infection. Those who endorsed either having fevers or having been told they had a bloodstream infection were asked whether: (1) the doctor indicated the symptoms/infection might be due to the PICC, (2) they had been admitted to the hospital, or (3) they had been prescribed antibiotics. Patients with a PICC still in place were also asked about function-related complications including difficulty with flushing or operating the PICC and restrictions in daily living or social activities. After completing the structured set of questions, patients were asked if they had any other problems with the PICC that had not been discussed; verbatim responses were recorded. Copies of the instruments are available in the online supplementary appendix 1 and online supplementary appendix 2 .

In addition to collecting patient-reported outcomes, research staff performed a review of medical records covering the same 70-day timeframe. Information collected included number of PICCs placed, number of lumens, insertion and removal dates and any documented occurrences of complications or signs and symptoms of a possible complication as included in the patient interview. Documentation of CLABSI or DVT was identified by an explicit statement of the condition by a medical provider in the notes section of the medical record.

\section{Study measures}

Medical outcomes of interest were reports by patients of a potential medical complication, as indicated by 
positive responses to the signs and symptoms described above that might indicate an infection, DVT or other minor issue like site redness. Functional outcomes while the PICC was in place included reports of difficulty with operating the PICC as well as those associated with quality of life such as activity and social restrictions. A summary measure of any reported complication, incorporating all outcomes, during the 70-day time frame was also created.

\section{Data analysis}

We calculated descriptive statistics for all variables of interest, including baseline patient characteristics (n $(\%)$, mean (SD)) and potential complications (n (\%)). The primary outcome was the percentage of patients reporting a potential PICC-related complication at any time during the 70-day follow-up period. We also assessed the percentage of patients who reported a potential complication at each assessment time point $(14,30,70$ days) based on whether the report occurred while the PICC was in place or removed. Additionally, we compared patient-reported responses of signs and symptoms suggestive of a bloodstream infection or venous thromboembolism with documentation of CLABSI or DVT in the medical record. All analyses were performed using Stata MP V.15.0 (College Station, TX).

\section{RESULTS}

From August 2015 through May 2017, a total of 1006 patients were identified as potentially eligible to participate in the study across the four hospitals. Of these, 623 were deemed eligible and $479(76.9 \%)$ provided written consent. A total of 41 participants were subsequently excluded due to missing data (either no initial survey or no response to the three follow-up surveys), resulting in an analytic data set of 438 patients $(91.4 \%$ of those consented for participation) (figure 1). Approximately $60 \%$ of patients $(n=264)$ responded to all three of the follow-up surveys.

On average, participating patients were 56 years of age. Two-thirds were male and the majority were white (79.2\%) (table 1). The most common reason for PICC placement, as reported by patients, was long-term antibiotic therapy (43.4\%), followed by chemotherapy $(20.5 \%)$ and difficult venous access (16.4\%). Most patients (79.2\%) reported that their PICC had been placed by a vascular access/PICC team nurse. About $17 \%$ of patients indicated that more than one placement attempt was required and nearly 30\% reported experiencing pain, discomfort, bleeding or other trauma during insertion. Over $80 \%$ of those participating had only one PICC during the 70-day follow-up period. Dwell time for the initial PICC was 5 days or less for about a quarter of participants and over 30 days for $30 \%$ of patients. Nearly $42 \%$ of patients had a single-lumen PICC, while $47.3 \%$ had a double-lumen device.

\section{Patient-reported medical complications}

During the 70-day follow-up period, $61.4 \%$ of participating patients reported at least one possible PICC-related complication or adverse effect. This included, as shown in table 2, signs and symptoms of potentially serious medical complications such as possible bloodstream infection $(n=77,17.6 \%)$ or DVT $(n=134,30.6 \%)$. Specifically, with respect to bloodstream infection, $12.1 \%$ of patients $(n=53)$ indicated having fever, chills or other symptoms suggestive of an infection that required them to see a doctor at one of the three follow-up time points. Approximately $40 \%$ of these patients reported a doctor indicated

Table 2 Medical complications up to 70 days after initial PICC placement

\begin{tabular}{|ll}
\hline & $\mathrm{n}(\%)$ \\
\hline Patient-reported signs and symptoms associated with possible bloodstream infection & $77 / 438(17.6)$ \\
\hline Fevers, chills or other symptoms suggestive of an infection that required you to see a doctor & $53 / 438(12.1)$ \\
\hline Doctor indicated might be due to an infection related to PICC or was admitted to the hospital & $23 / 53(43.4)$ \\
\hline Prescribed antibiotics & $38 / 53(71.7)$ \\
\hline Were you told you have a bloodstream infection ( $\mathrm{n}=387)^{*}$ & $31 / 387(8.0)$ \\
\hline Doctor indicated might be due to an infection related to PICC or was admitted to the hospital & $24 / 31(77.4)$ \\
\hline Prescribed antibiotics & $27 / 31(87.1)$ \\
\hline Documentation of central line-associated bloodstream infection in medical record & $7 / 438(1.6)$ \\
\hline Patient-reported symptoms associated with possible deep vein thrombosis & $134 / 438(30.6)$ \\
\hline Redness, pain or swelling in the hand, arm or shoulder in the arm where the line was inserted & $57 / 438(13.0)$ \\
\hline Chest pain, shortness of breath or difficulty breathing that required you to seek emergency care & $37 / 438(8.4)$ \\
\hline Pain, swelling, redness or tenderness in either of your legs or calves & $80 / 438(18.3)$ \\
Deep vein thrombosis documented in medical record & $31 / 438(7.1)$ \\
Patient-reported symptoms suggestive of minor complications & $122 / 438(27.9)$ \\
\hline Redness around insertion site & $95 / 438(21.7)$ \\
\hline Discomfort, inadvertent removal, migration or difficulty when removed & $37 / 438(8.4)$ \\
\hline
\end{tabular}

PICC, peripherally inserted central catheter.

${ }^{*}$ Asked only of those with PICC removed. 
their symptoms might be due to an infection related to the PICC or being admitted to the hospital; more than $70 \%$ reported receiving antibiotics. Among patients whose PICC had been removed, $8.0 \%(n=31)$ indicated they were told they had a bloodstream infection; 24 of whom reported a doctor indicated the infection might be related to the PICC or admission to a hospital and 27 (87.1\%) were prescribed antibiotics. Although CLABSI was documented in the medical record for seven patients, only three were also part of the group of 77 patients who reported signs and symptoms of an infection or being told they had a bloodstream infection.

Of the 134 patients reporting signs of a possible DVT, $13.0 \%$ reported redness, pain or swelling in the arm where the line was inserted, $8.4 \%$ chest pain or shortness of breath requiring them to seek care and $18.3 \%$ had pain, swelling or redness in their lower extremities. Documentation of DVT was identified in the medical record for 31 patients. However, only 14 of these patients were among the group of 134 who reported symptoms of a possible DVT.

In addition to signs and symptoms of CLABSI and DVT, more than one-quarter $(27.9 \%)$ of patients reported experiencing at least one minor complication. In particular, $21.7 \%$ noted redness around the insertion site, both while the PICC was in place and after removal. Other commonly described issues were discomfort, inadvertent removal, migration or a difficult removal (8.4\%).

\section{Change in patient-reported complications over time} Longitudinal analyses of the patient-reported signs and symptoms showed differing trends of patient-reported complications. For example, while the PICC was in place (figure $2 \mathrm{~A}$ ), the percentage of patients reporting issues such as insertion site redness and upper extremity redness, pain or swelling decreased over time. On the other hand, the percentage reporting signs of potential infection (ie, fever, chills) increased slightly from the 14-day follow-up (7.3\%) to the 30-day follow-up (8.4\%), while the percentage experiencing lower extremity pain, swelling or redness showed a more substantial increase from $5.2 \%$ at 14 days to $12.3 \%$ at 30 days. Likewise, nearly $14 \%$ of the 37 patients with a PICC in place at the 70-day follow-up reported lower extremity pain, swelling and redness, raising concern about possible DVT. Among patients with the PICC removed (figure $2 \mathrm{~B}$ ), the percentage reporting site redness decreased over time (from $13.6 \%$ at 14 days to $10.0 \%$ at 70 days).

\section{Patient-reported function and experiences while PICC is in place}

In addition to potential medical complications, patients also reported other types of complications or adverse effects while the PICC was in place (table 3). Specifically, more than a quarter of patients with a PICC in place $(26.0 \%)$ reported restrictions in activities of daily living, $14.4 \%$ noted social activity restrictions and $19.2 \%$ had difficulty with flushing or operating the PICC. Concerns related to these limitations were also evident in some of the unsolicited comments by patients, such as: 'It's just annoying that it's there, I have to be careful when taking showers and doing other activities. It hurts from time to time.' Some patients, on the other hand, appreciated the convenience of having a PICC: 'It's convenient, better than getting poked all the time.'

\section{Discussion}

While PICCs remain an important part of medical care, our assessment of patient-reported complications across a diverse patient population provides novel and important insights about potential harm from these devices. For example, we found that over $60 \%$ of patients reported experiencing at least one potential complication related to their PICC. In addition to signs or symptoms associated with certain medical complications, patients reported challenges related to PICC use as well as activity and social restrictions. Moreover, nearly $30 \%$ of patients reported pain, discomfort, bleeding or trauma on insertion-a surprisingly high percentage. Our findings also demonstrate that patient-reported outcomes can serve as a complement to traditionally reported metrics, which are primarily based on medical record data, to help identify further opportunities for mitigating PICC-related complications and inform programmes designed to improve the patient experience for those with PICCs.

The fact that PICCs are associated with serious medical complications, such as CLABSI and DVT, is well established. ${ }^{7} 1819$ The estimated frequency of these events among adult patients in the USA generally ranges from $<1 \%$ to $6 \%$ for bloodstream infection, ${ }^{9102021}$ and excluding studies that involve screening for thrombosis from $2 \%$ to $15 \%$ for DVT. ${ }^{10} 1822$ While the estimates vary, depending on study population (eg, patients with cancer, the critically ill, outpatients) and across sites, our findings also suggest that the frequency of these potentially serious complications may in some circumstances be underestimated without systematic patient follow-up. For example, while 31 patients indicated being told they have a bloodstream infection (of which nine also reported being told it might be due to the PICC), documentation in the medical record of a CLABSI was found for only three of these patients. Although these discrepancies may reflect infections not related to the PICC or misattribution of patient-reported symptoms, it is equally plausible that medical record data from the institution where the PICC was placed underestimate the true rate of PICC complications. Furthermore, given the potentially serious nature of these types of complications and frequency of certain symptoms, our findings indicate that patients need to be prepared on 


\section{A}

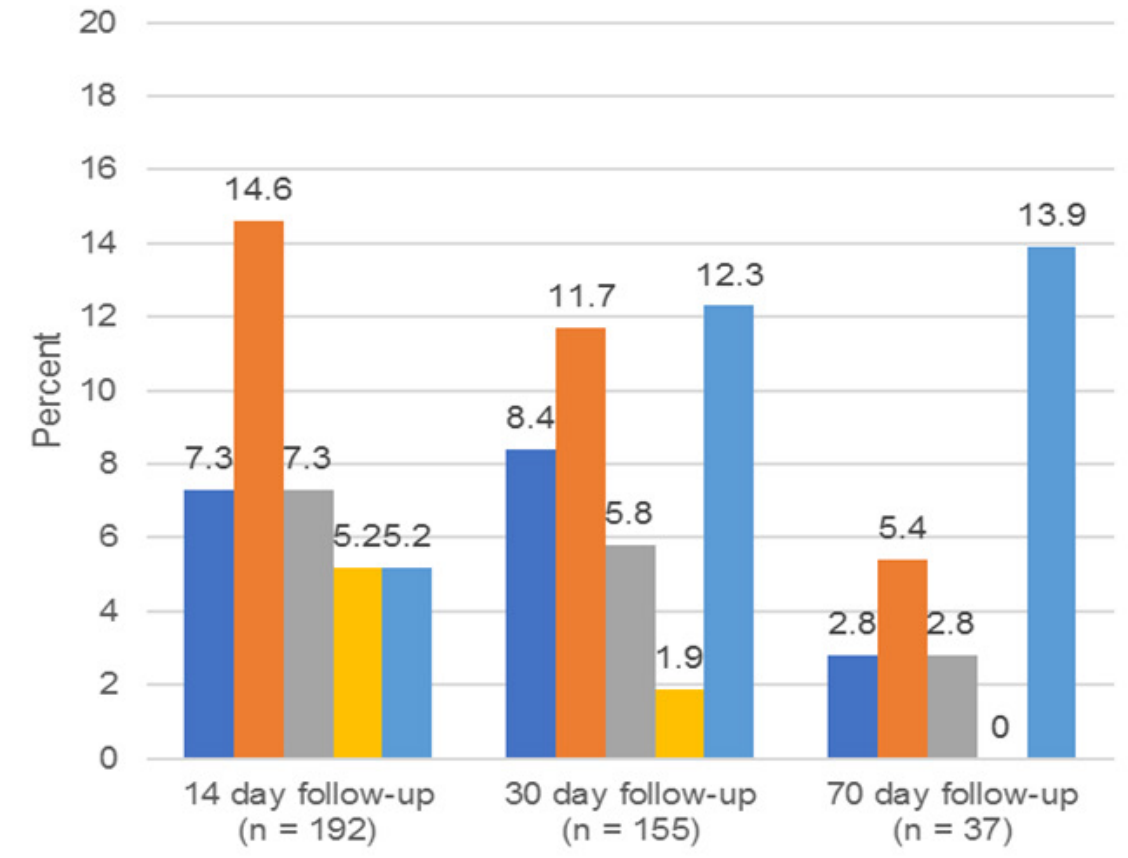
- Fever, chills, symptoms of infection

n Site redness

- Upper extremity

redness, pain, swelling

EChest pain, shortness of breath or difficulty breathing

- Lower extremity pain, swelling, redness

\section{B}

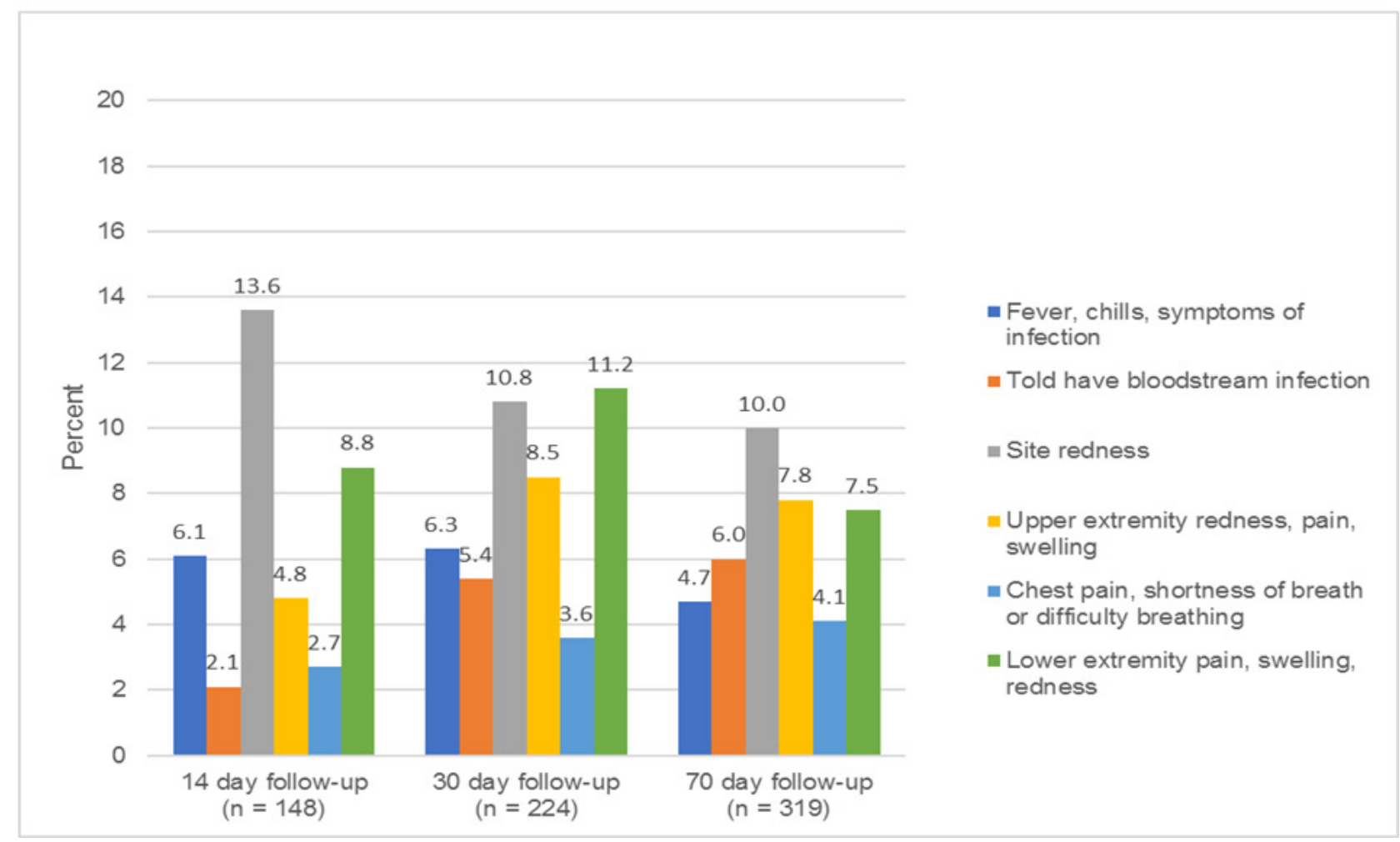

Figure 2 (A) Patient-reported medical complications while peripherally inserted central catheter (PICC) is in place (by type, at each reporting time point). (B) Patient-reported medical complications with PICC removed (by type, at each reporting time point).

the specific follow-up actions to take when signs or symptoms of CLABSI or DVT occur.

Despite growing use, little is known about how a PICC influences quality of life and activities of daily living. ${ }^{14-16} 23$ Available data suggest that while patients are apprehensive at first, they tend to adapt to living with a PICC, ${ }^{16} 23$ and for many, the experience is viewed as positive. ${ }^{141623}$ On the other hand, 
Table 3 Patient-reported function and experiences while PICC was in place

\begin{tabular}{l}
\hline \\
\hline Restrictions in activities of daily living related to having $65 / 250$ (26.0) \\
the catheter \\
Restrictions in social activities related to having the $36 / 250$ (14.4) \\
catheter \\
Difficulty flushing, accessing or otherwise operating the \\
PICC \\
Example comments: \\
'It's just annoying that it's there, I have to be careful when taking \\
showers and doing other activities. It hurts from time to time.' \\
'When he showers, there's no simple easy way to seal the PICC line up \\
- doesn't understand how we can send people to the moon, but can't \\
invent a sleeve to keep arm dry.' \\
'PICC is placed in right arm above elbow, gets in the way of her doing \\
things.' \\
'PICC allowing fluid to go in, but won't draw back or allow blood to \\
come out, so any blood draws can't use PICC line, have to use other \\
needle.' \\
'It seems to be functioning well, I have not had any problems. It's great \\
to have it in.' \\
'It's convenient, better than getting poked all the time.' \\
\hline PICC, peripherally inserted central catheter.
\end{tabular}

PICC, peripherally inserted central catheter.

the experience is substantially less positive for those who experience a PICC-related complication. ${ }^{15} 24$ Our findings add to what is known by providing data on how many patients experience some degree of negative impact during PICC use, with more than a quarter of patients endorsing restrictions in activities of daily living and 14\% social activity restrictions. Moreover, almost one in five patients experienced challenges with operating their PICC, including flushing and accessing the device. Several patients also described issues with PICC dressings, including 'itchiness'. Although these types of complications may not be viewed as critical from a medical perspective, they are important to patients and can lead to unnecessary anxiety or even additional healthcare utilisation. The complications that receive serious attention from healthcare providers are likely only the tip of the iceberg of all bothersome or anxiety-producing adverse effects associated with PICCs, from the patient perspective. As such, well-defined strategies for ensuring that patients are properly monitored, informed and equipped to deal with not only potential medical complications but how their lives will be affected by having a PICC are clearly needed.

Our study has several limitations. First, our assessment of patient-reported complications primarily relied on patients answering a structured set of questions regarding certain signs and symptoms. Although these questions specifically asked about issues related to PICCs, this strategy may have resulted in patients reporting symptoms (eg, chest pain and shortness of breath) related to another underlying condition. However, whether related to the PICC or not, our study shows that such symptoms are of concern for patients and suggests a need for further investigation to ensure that these issues are appropriately addressed. Second, we did not collect data about patient comorbidities or patient literacy, issues that could potentially influence the rate of reported complications; we thus cannot adjust for these factors in our analysis. Third, because some patients likely received follow-up care at non-study facilities, we are not able to verify all patient-reported occurrences of a possible CLABSI or DVT as we did not obtain medical records from these sites. Finally, although we report study findings in aggregate, site-specific differences in patient-reported complications were also observed, likely reflecting local practices and types of patients treated.

Our study also has several strengths. To our knowledge, this is the first large-scale study focusing on patient-reported PICC complications from a diverse sample of patients who received care at different hospitals. Second, we followed patients prospectively over an extended 70-day period regardless of PICC presence to identify medical and non-medical complications and assess how signs and symptoms vary over time. Third, this study demonstrates the feasibility of collecting patient-reported outcomes and the utility of going beyond the hospital setting to better understand, target and alleviate PICC-related complications.

Although increasing PICC use highlights the importance of these devices for delivering important medical therapies, ensuring safe and appropriate use remains paramount. Our findings reveal that more than $60 \%$ of patients report experiencing signs and symptoms of at least one PICC-related complication or adverse effect, including challenges with PICC use and negative effects on physical and social function while living with a PICC. Bothersome complications from the patient perspective are clearly more common than those that typically rise to the level of healthcare provider attention or concern. Understanding the patient experience is therefore a key step in not only developing strategies to better monitor PICC-related complications, but perhaps more importantly for providing patients with the support they need to ensure safe, effective patient-centred care.

\section{Author affiliations}

${ }^{1}$ Center for Clinical Management Research, VA Ann Arbor Healthcare System, Ann Arbor, Michigan, USA

${ }^{2}$ Division of General Medicine, Department of Internal Medicine, University of Michigan Medical School, Ann Arbor, Michigan, USA

${ }^{3}$ Division of Hospital Medicine, Department of Internal Medicine, University of Michigan Medical School, Ann Arbor, Michigan, USA

${ }^{4}$ Center for Innovations in Quality, Effectiveness and Safety, Michael E. DeBakey Veterans Affairs Medical Center, Houston, Texas, USA

${ }^{5}$ Department of Internal Medicine, Baylor College of Medicine, Houston, Texas, USA

Contributors The authors thank the following individuals who assisted with data collection and other study-related activities: Laura Dillon, Jeanaya McKinley, Laura Peña, Jason Mann, Marylena Rouse, Kathy Swalwell, Suzanne Winter, Jane Wong, 
Debbie Zawol and Karen Fowler. They also thank the patients who so graciously participated and provided data for this study.

Funding This study was funded by the US Department of Veterans Affairs (VA) Health Services Research and Development Service (grant numbers IIR 12-395 and RCS 11-222) with additional support provided by the VA National Center for Patient Safety through a Patient Safety Center of Inquiry.

Disclaimer The views expressed in this article are those of the authors and do not necessarily reflect the position or policy of the Department of Veterans Affairs or the United States government.

Competing interests None declared.

Patient consent for publication Not required.

Ethics approval The institutional review board approval was received from each participating hospital: VA Ann Arbor Healthcare System, University of Michigan Medical School IRB, Baylor College of Medicine IRB and Harris Health.

Provenance and peer review Not commissioned; externally peer reviewed.

Data sharing statement Deidentified data will be made available after all ongoing analyses are completed. Requesters will be required to sign a Letter of Agreement detailing the mechanisms by which the data will be kept secure and access restricted to their study team. The agreements will also state the recipient will not attempt to identify any individual whose data are included and will not share the data with anyone outside of their research team. The data set will not include PII and all dates removed.

Open access This is an open access article distributed in accordance with the Creative Commons Attribution Non Commercial (CC BY-NC 4.0) license, which permits others to distribute, remix, adapt, build upon this work noncommercially, and license their derivative works on different terms, provided the original work is properly cited, appropriate credit is given, any changes made indicated, and the use is noncommercial. See: http://creativecommons.org/licenses/by-nc/4.0

\section{REFERENCES}

1 Hoshal VL. Total intravenous nutrition with peripherally inserted silicone elastomer central venous catheters. Arch Surg 1975;110:644-6.

2 Gow KW, Tapper D, Hickman RO. Between the lines: the 50th anniversary of long-term central venous catheters. Am J Surg 2017;213:837-48.

3 Chopra V, Kuhn L, Ratz D, et al. Vascular access specialist training, experience, and practice in the United States: results from the national PICC1 survey. J Infus Nurs 2017;40:243-9.

4 Horattas MC, Trupiano J, Hopkins S, et al. Changing concepts in long-term central venous access: catheter selection and cost savings. Am J Infect Control 2001;29:32-40.

5 Chopra V, Flanders SA, Saint S. The problem with peripherally inserted central catheters. JAMA 2012;308:1527-8.

6 McMahon LF, Beyth RJ, Burger A, et al. Enhancing patientcentered care: SGIM and choosing wisely. J Gen Intern Med 2014;29:432-3.

7 Chopra V, Anand S, Hickner A, et al. Risk of venous thromboembolism associated with peripherally inserted central catheters: a systematic review and meta-analysis. Lancet 2013;382:311-25.
8 Chopra V, Anand S, Krein SL, et al. Bloodstream infection, venous thrombosis, and peripherally inserted central catheters: reappraising the evidence. Am J Med 2012;125:733-41.

9 Chopra V, O'Horo JC, Rogers MA, et al. The risk of bloodstream infection associated with peripherally inserted central catheters compared with central venous catheters in adults: a systematic review and meta-analysis. Infect Control Hosp Epidemiol 2013;34:908-18.

10 Chopra V, Smith S, Swaminathan L, et al. Variations in peripherally inserted central catheter use and outcomes in michigan hospitals. JAMA Intern Med 2016;176:548-51.

11 O'Brien C, McMorrow J, O'Dwyer E. Peripherally Inserted Central Catheters (PICCs) and potential cost savings and shortened bed stays in an acute hospital setting. Ir Med J 2018;111:670.

12 Chopra V, Montoya A, Joshi D, et al. Peripherally inserted central catheter use in skilled nursing facilities: a pilot study. J Am Geriatr Soc 2015;63:1894-9.

13 Harrod M, Montoya A, Mody L, et al. Challenges for nurses caring for individuals with peripherally inserted central catheters in skilled nursing facilities. J Am Geriatr Soc 2016;64:2059-64.

14 Parás-Bravo P, Paz-Zulueta M, Santibañez M, et al. Living with a peripherally inserted central catheter: the perspective of cancer outpatients-a qualitative study. Support Care Cancer 2018;26:441-9.

15 Meyer BM. Understanding the patient experience of peripherally inserted central catheter-related deep vein thrombosis using interpretive phenomenology. J Infus Nurs 2017;40:287-96.

16 Sharp R, Grech C, Fielder A, et al. The patient experience of a peripherally inserted central catheter (PICC): a qualitative descriptive study. Contemp Nurse 2014;48:26-35.

17 Greene MT, Flanders SA, Woller SC, et al. The association between PICC use and venous thromboembolism in upper and lower extremities. Am J Med 2015;128:986-93.

18 Fallouh N, McGuirk HM, Flanders SA, et al. Peripherally inserted central catheter-associated deep vein thrombosis: a narrative review. Am J Med 2015;128:722-38.

19 Ajenjo MC, Morley JC, Russo AJ, et al. Peripherally inserted central venous catheter-associated bloodstream infections in hospitalized adult patients. Infect Control Hosp Epidemiol 2011;32:125-30.

20 Moran J, Colbert CY, Song J, et al. Screening for novel risk factors related to peripherally inserted central catheterassociated complications. J Hosp Med 2014;9:481-9.

21 Chopra V, Ratz D, Kuhn L, et al. PICC-associated bloodstream infections: prevalence, patterns, and predictors. Am J Med 2014;127:319-28.

22 Cotogni P, Barbero C, Garrino C, et al. Peripherally inserted central catheters in non-hospitalized cancer patients: 5 -year results of a prospective study. Support Care Cancer 2015;23:403-9.

23 Harrold K, Martin A, Scarlett C. Proactive PICC placement: evaluating the patient experience. Br J Nurs 2016;25:S4-14.

24 Gao W, Luan XR, Sun YY, et al. Experiences of patients with abnormal extubation of PICC tubes: a qualitative study. Int $J$ Clin Exp Med 2015;8:19297-303. 